\title{
Antenatal Corticosteroids for Pregnant Women at High Risk of Preterm Delivery with COVID-19 Infection: A Decision Analysis
}

\author{
Claire H. Packer, BA ${ }^{1}$ Clarice G. Zhou, MD ${ }^{1}$ Alyssa R. Hersh, MD, MPH ${ }^{1}$ Allison J. Allen, MD ${ }^{1}$ \\ Amy C. Hermesch, MD, $\mathrm{PhD}^{1}$ Aaron B. Caughey, $\mathrm{MD}, \mathrm{PhD}^{1}$ \\ ${ }^{1}$ Department of Obstetrics and Gynecology, Oregon Health and
Science University, Portland, Oregon \\ Am J Perinatol 2020;37:1015-1021. \\ Address for correspondence Claire H. Packer, BA, Department of \\ Obstetrics and Gynecology, Oregon Health and Science University, \\ 3181 Southwest Sam Jackson Park Road, Portland, OR 97339-3908 \\ (e-mail: packerc@ohsu.edu).
}

Keywords

- preterm birth

- COVID-19

- antenatal steroids

- respiratory infection
Objective Antenatal corticosteroids given prior to preterm deliveries reduce the risk of adverse neonatal outcomes. However, steroid administration in the setting of a viral respiratory infection can worsen maternal outcomes. Therefore, the decision to administer corticosteroids must balance the neonatal benefits with the potential harm to the mother if she is infected with the novel coronavirus disease 2019 (COVID19). This study aimed to determine the gestational ages for which administering antenatal corticosteroids to women at high risk of preterm labor with concurrent COVID-19 infection results in improved combined maternal and infant outcomes.

Study Design A decision-analytic model using TreeAge (2020) software was constructed for a theoretical cohort of hospitalized women with COVID-19 in the United States. All model inputs were derived from the literature. Outcomes included maternal intensive care unit (ICU) admission and death, along with infant outcomes of death, respiratory distress syndrome, intraventricular hemorrhage, and neurodevelopmental delay. Quality-adjusted life years (QALYs) were assessed from the maternal and infant perspectives. Sensitivity analyses were performed to determine if the results were robust over a range of assumptions. Results In our theoretical cohort of 10,000 women delivering between 24 and 33 weeks of gestation with COVID-19, corticosteroid administration resulted in 2,200 women admitted to the ICU and 110 maternal deaths. No antenatal corticosteroid use resulted in 1,500 ICU admissions and 75 maternal deaths. Overall, we found that corticosteroid administration resulted in higher combined QALYs up to 31 weeks of gestation in all hospitalized patients, and up to 29 weeks of gestation in ICU patients. Conclusion Administration of antenatal corticosteroids at less than 32 weeks of gestation for hospitalized patients and less than 30 weeks of gestation for patients admitted to the ICU resulted in higher combined maternal and infant outcomes compared with expectant management for women at high risk of preterm birth with COVID-19 infection. These results can guide clinicians in their counseling and management of these pregnant women.

\section{Key Points}

- Antenatal steroids reduce adverse neonatal outcomes.

- Steroids worsen maternal outcomes in COVID-19.

- Steroids given $<32$ weeks result in improved outcomes.

received

May 15,2020

accepted after revision

May 15, 2020

published online

June 30,2020
Copyright $\odot 2020$ by Thieme Medical Publishers, Inc., 333 Seventh Avenue, New York, NY 10001, USA. Tel: +1(212) 760-0888.
DOI https://doi.org/

10.1055/s-0040-1713145. ISSN 0735-1631. 
Shortly following the emergence of the novel coronavirus disease 2019 (COVID-19) several guidelines were published regarding the management of pregnant women and their infants. ${ }^{1-3}$ The first data emerged from the experience of health care providers in Wuhan, China, and knowledge of COVID-19 continues to increase as the pandemic spreads. ${ }^{4}$ It remains unclear if use of antenatal corticosteroids for fetal lung maturation and decreasing neonatal morbidity and mortality outweighs the maternal risk of corticosteroid exposure in women with COVID-19 infection.

When a woman is at high risk of preterm birth, corticosteroids are administered to decrease risk of neonatal morbidity associated with prematurity. According to a 2017 Cochrane review, antenatal corticosteroid administration is associated with reduced neonatal death, respiratory distress syndrome (RDS), and intraventricular hemorrhage (IVH), among other improved clinical outcomes. ${ }^{5}$ Although there were limited number of studies included in the analysis on maternal morbidity and mortality, it was concluded that there was no increased risk of maternal harm from antenatal corticosteroid exposure.

During the severe acute respiratory syndrome-coronavirus (SARS-CoV) and Middle East respiratory syndrome-CoV (MERS-CoV) outbreaks, steroids were commonly used to manage hospitalized patients. ${ }^{6}$ However, studies following the outbreaks demonstrated corticosteroid therapy was associated with worse outcomes, including increased mortality and delayed clearance of viral RNA. ${ }^{7-10}$ Given the evidence available from previous outbreaks, corticosteroid therapy is currently not routinely recommended for use in patients with COVID-19 infection.

This, however, presents a clinical dilemma for obstetrics patients, for whom the health of both the woman and infant are important in medical decision making. While women at high risk of preterm birth are typically managed with antenatal corticosteroids for infant benefit, clinicians must weigh the potential harms associated with corticosteroid use in a patient with COVID-19 infection. Therefore, we sought to determine the gestational ages for which administration of antenatal corticosteroids to women at high risk of preterm birth with COVID-19 infection results in higher combined maternal and infant outcomes than expectant management.

\section{Materials and Methods}

We created a decision-analytic model using TreeAge (2020 version, TreeAge Software Inc., Williamstown, MA,) software to assess the clinical outcomes and total maternal and infant quality-adjusted life years (QALYs) of the administration of antenatal corticosteroids for women at high risk of preterm birth with COVID-19 infection. We used a theoretical cohort of 10,000 women in the United States who were infected with COVID-19 and also at high risk of preterm birth at each gestational age between $24^{0 / 7}$ and $33^{6 / 7}$ weeks. This theoretical cohort was used as the COVID-19 pandemic continues to evolve with estimates of potential impact on women and pregnancy changing daily, so a more precise theoretical cohort was not calculated. As this was a theoretical model with inputs derived from the published literature, and no patients were required for the study, the study was deemed to be exempt from Institutional Review Board (IRB) approval.

The model begins by separating the two strategies: administration of antenatal corticosteroids versus expectant management with no administration of corticosteroids ( - Fig. 1). It was assumed that all women delivered during the gestational age in which they were given steroids. Antenatal corticosteroid treatment consists of two injections of betamethasone given 24 hours apart to improve fetal lung maturity in preterm deliveries if clinical indications were consistent with imminent delivery within 7 days. Then, women were stratified based on whether they needed intensive care unit (ICU) admission or died. Infant outcomes of interest were death, RDS, IVH, and neurodevelopmental delay.

Our probabilities and utilities were derived from literature and these model inputs are shown in - Table 1. The probabilities for maternal ICU admission and death from COVID-19 infection were estimated from the reported cases in the United States of individuals aged 20 to 44 years up to March 16, 2020 by the Centers for Disease Control and Prevention (CDC) COVID-19 response team. ${ }^{11}$ The effects of steroids on probability of admission to ICU and mortality was estimated by a hazard ratio of 1.49 that was reported in a systematic review of steroids in the setting of hospitalized patients with influenza. ${ }^{12}$ In hospitalized patients, we assumed a probability of 0.22 for ICU admission with steroid administration, and 0.15 for ICU admission without steroids. We assumed that if a woman with COVID-19 was to die, she would have been previously admitted to the ICU. We utilized a probability of maternal death of 0.05 for women treated with steroids or not, as her probability of being admitted to the ICU was affected by steroid use. However, for our model of women already in the ICU, we assumed her probability of death with steroid administration was 0.075 and 0.05 without steroids. ${ }^{11}$

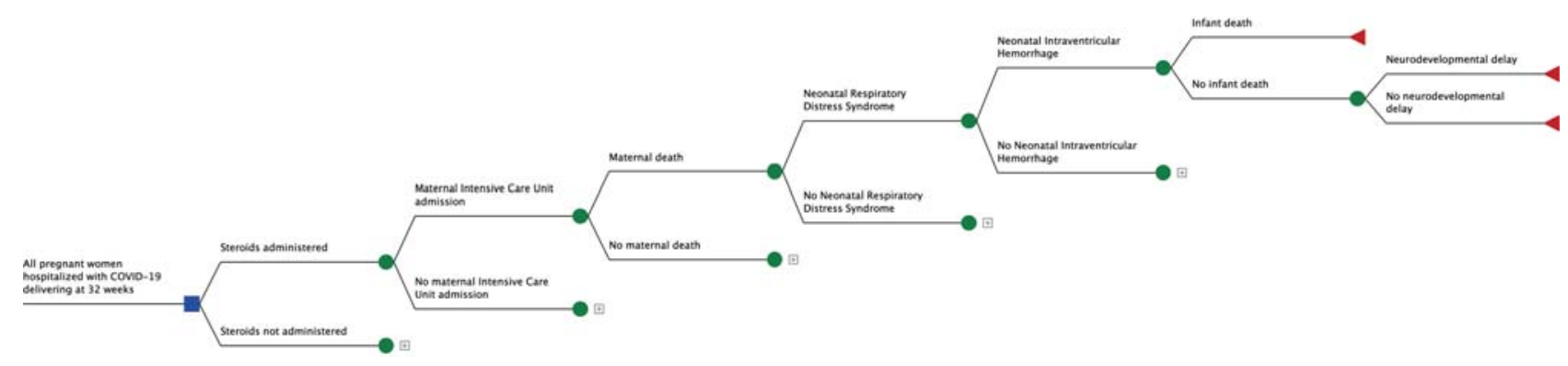

Fig. 1 Decision-analytic model schematic. 
Table 1 Common model inputs

\begin{tabular}{|c|c|c|}
\hline Parameter & $\begin{array}{l}\text { Baseline } \\
\text { input }\end{array}$ & $\begin{array}{l}\text { Reference } \\
\text { number }\end{array}$ \\
\hline \multicolumn{3}{|l|}{ Probabilities } \\
\hline Maternal ICU admission & & 12 \\
\hline Steroids & 0.22 & \\
\hline No steroids & 0.15 & \\
\hline $\begin{array}{l}\text { Maternal death, hospitalized } \\
\text { patients }\end{array}$ & & 11 \\
\hline Steroids & 0.05 & \\
\hline No steroids & 0.05 & \\
\hline Maternal death, ICU patients & & 11,12 \\
\hline Steroids & 0.075 & \\
\hline No steroids & 0.05 & \\
\hline \multicolumn{3}{|l|}{ Utilities } \\
\hline Healthy & 1.00 & Assumed \\
\hline Maternal ICU admission & 0.66 & 22 \\
\hline $\begin{array}{l}\text { Neonatal ICU admission, } \\
\text { maternal perspective }\end{array}$ & 0.95 & 23 \\
\hline \multicolumn{3}{|l|}{$\begin{array}{l}\text { Neonatal } \\
\text { Neurodevelopmental Delay }\end{array}$} \\
\hline Maternal perspective & 0.76 & 23 \\
\hline Infant perspective & 0.69 & 25 \\
\hline $\begin{array}{l}\text { Infant death, maternal } \\
\text { perspective }\end{array}$ & 0.92 & 24 \\
\hline \multicolumn{3}{|l|}{ Life expectancies } \\
\hline \multicolumn{3}{|l|}{ Healthy with NICU stay } \\
\hline Maternal & 56.2 & 18,20 \\
\hline Infant & 78.8 & 20 \\
\hline NDD & 62.0 & 21 \\
\hline NICU stay & 0.078 & 26 \\
\hline ICU stay effect duration & 1 & 22 \\
\hline
\end{tabular}

Abbreviations: ICU, intensive care unit; NDD, neurodevelopmental delay; NICU, neonatal ICU.

For infant outcomes, the probabilities of RDS, IVH and death by gestational age from 24 to 33 weeks were determined from a large prospective cohort study that compared antenatal corticosteroid administration to no treatment ( - Supplementary Table S1, available in the online version). ${ }^{5,13}$ The probability of neurodevelopmental delay was estimated from a prospective cohort study, which assessed cognitive disability and hearing and visual impairment of children born before 33 weeks of gestation at 5 years of age ( - Supplementary Table S2, available in the online version). ${ }^{14}$ Neurodevelopmental sequelae and infant death differences with presence or absence of IVH were calculated based on an odds ratio of 6.02 and 5.64, respectively. ${ }^{15}$

Utilities were included from both the maternal and infant perspective and discounted at an annual rate of $3 \%{ }^{16}$ Utilities are used to estimate the quality of life and range from 0 to 1 , with 0 being death and 1 meaning perfect health. Utilities are then applied to discounted life-years to generate discounted quality-adjusted life years (QALYs). A woman's life expectancy following a first pregnancy was estimated to be 56.2 years based on average age of first birth and current estimated life expectancy. ${ }^{17,18}$ For healthy children and neurodevelopmentally delayed children, life expectancies were estimated to be 78.8 years and 62 years, respectively. ${ }^{19-21}$

For any woman who required ICU admission, a utility of 0.66 was used and applied for 1 year, based on quality of life estimates for 1 year after initial ICU admission. ${ }^{22}$ Utilities regarding infant outcomes from the maternal perspective were derived from data from Carroll and Downs which asked guardians to provide utilities for chosen health states that included having a child in perfect health, a child who died, and a child with neurodevelopmental delay. ${ }^{23}$ We estimated the maternal utility for having a child with neurodevelopmental delay by using the value for moderate cerebral palsy, 0.76 . We estimated a maternal utility of 0.92 for having an infant who did not survive. ${ }^{24}$

For infant utilities, we used survey data, which assessed selfperceived quality of life of extremely low birth weight neonates who were followed into adulthood. The infant utility of moderate neurodevelopmental delay was estimated to be 0.69 . In addition, as all neonates born in this model were preterm, we applied a utility of 0.95 for the average length of stay for preterm neonate, which we calculated to be 28.3 days. ${ }^{23,25,26}$

Sensitivity analyses were performed on all model inputs to determine the robustness of the results. We performed a tornado analysis, which is a mechanism of running one-way sensitivity analyses on all model inputs simultaneously to determine which inputs have the most effect on the model. Model inputs identified then were subjected to focused oneway sensitivity analyses to determine thresholds.

We also performed probabilistic sensitivity analysis via Monte Carlo simulation, which enabled us to incorporate variation into all model inputs simultaneously and determine the proportion of the time a strategy is still effective with 1,000 samples. Beta distributions were used for probabilities and utilities, which approximate the normal distribution and cannot fall outside of the range 0 to 1 . Triangular distributions were used for life expectancies. The results of the simulation are presented in a bar graph, showing the confidence of each strategy.

\section{Results}

In our theoretical cohort of 10,000 women at high risk of preterm delivery between 24 and 33 weeks of gestation with COVID-19 infection, corticosteroid administration resulted in 2,200 women admitted to the ICU and 110 maternal deaths at each gestational age ( - Table 2 ). In contrast, no antenatal corticosteroids resulted in 1,500 ICU admissions and 75 maternal deaths at each gestational age. Of the women already admitted into the ICU, there were 500 deaths without steroid administration and 745 deaths with steroids (-Table 3). Although administration of corticosteroids resulted in worse maternal morbidity and mortality for women, it was associated with better outcomes for infants, including fewer cases of RDS, IVH, and death. For example, at 
Table 2 Outcomes in a theoretical cohort of 10,000 women hospitalized with COVID-19 and at risk of preterm delivery at 24, $28,31,32$, and 33 weeks of gestation

\begin{tabular}{|c|c|c|c|c|c|c|c|c|c|c|}
\hline & \multicolumn{2}{|c|}{24 weeks } & \multicolumn{2}{|c|}{28 weeks } & \multicolumn{2}{|c|}{31 weeks } & \multicolumn{2}{|c|}{32 weeks } & \multicolumn{2}{|c|}{33 weeks } \\
\hline & Steroids & $\begin{array}{l}\text { No } \\
\text { steroids }\end{array}$ & Steroids & $\begin{array}{l}\text { No } \\
\text { steroids }\end{array}$ & Steroids & $\begin{array}{l}\text { No } \\
\text { steroids }\end{array}$ & Steroids & $\begin{array}{l}\text { No } \\
\text { steroids }\end{array}$ & Steroids & $\begin{array}{l}\text { No } \\
\text { steroids }\end{array}$ \\
\hline \multicolumn{11}{|l|}{ Maternal outcomes } \\
\hline ICU admission & 2,220 & 1,500 & 2,220 & 1,500 & 2,200 & 1,500 & 2,220 & 1,500 & 2,220 & 1,500 \\
\hline Death & 110 & 75 & 110 & 75 & 110 & 75 & 110 & 75 & 110 & 75 \\
\hline \multicolumn{11}{|l|}{ Infant outcomes } \\
\hline $\begin{array}{l}\text { Respiratory distress } \\
\text { syndrome }\end{array}$ & 2,488 & 3,740 & 1,060 & 1,610 & 410 & 620 & 280 & 420 & 190 & 290 \\
\hline $\begin{array}{l}\text { Intraventricular } \\
\text { hemorrhage }\end{array}$ & 1,760 & 2,610 & 300 & 530 & 60 & 90 & 30 & 40 & 10 & 20 \\
\hline $\begin{array}{l}\text { Neurodevelopmental } \\
\text { delay }\end{array}$ & 1,209 & 867 & 1,386 & 1,374 & 757 & 757 & 415 & 415 & 123 & 124 \\
\hline Death & 4,303 & 6,059 & 369 & 787 & 51 & 135 & 30 & 61 & 10 & 30 \\
\hline
\end{tabular}

Abbreviations: COVID-19, novel coronavirus disease 2019; ICU, intensive care unit.

Table 3 Outcomes in a theoretical cohort of 10,000 women admitted to the ICU with COVID-19 and at risk of preterm delivery at 29,30 , and 31 weeks of gestation

\begin{tabular}{|c|c|c|c|c|c|c|}
\hline & \multicolumn{2}{|c|}{29 weeks } & \multicolumn{2}{|c|}{30 weeks } & \multicolumn{2}{|c|}{31 weeks } \\
\hline & Steroids & No steroids & Steroids & No steroids & Steroids & No steroids \\
\hline \multicolumn{7}{|l|}{ Maternal outcomes } \\
\hline Death & 745 & 500 & 745 & 500 & 745 & 500 \\
\hline \multicolumn{7}{|l|}{ Infant outcomes } \\
\hline Respiratory distress syndrome & 770 & 1,180 & 500 & 760 & 410 & 620 \\
\hline Intraventricular hemorrhage & 150 & 310 & 100 & 220 & 60 & 90 \\
\hline Neurodevelopmental delay & 1,238 & 1,250 & 919 & 942 & 783 & 768 \\
\hline Death & 191 & 414 & 125 & 208 & 52 & 134 \\
\hline
\end{tabular}

Abbreviations: COVID-19, novel coronavirus disease 2019; ICU, intensive care unit.

28 weeks of gestation, corticosteroid administration resulted in 550 fewer cases of RDS, 230 fewer cases of IVH and 419 fewer deaths.

Total QALYs are shown in - Table 4. We found that management with antenatal corticosteroids in hospitalized patients with COVID-19 infection resulted in higher QALYs from a combined maternal and infant perspective from $24^{0 / 7}$ to 31 ${ }^{6 / 7}$ weeks of gestation ( - Table 4; - Supplementary Tables S3s10 [available in the online version]). Despite the worse maternal outcomes with corticosteroid use at 28 weeks of gestation and greater, the infant benefits of steroids below 32 weeks of gestation outweighed the potential maternal harm. At 32 and 33 weeks of gestation, management without antenatal corticosteroid administration resulted in higher QALYs and thus was the optimal strategy (-Supplementary Tables S11 and S12, available in the online version).

Among women in the ICU due to COVID-19 infection, there was higher risk of maternal death with corticosteroid administration. Due to this, antenatal steroid administration was the optimal strategy through 29 weeks of gestation, resulting in higher QALYs than expectant management. At
30 weeks of gestation, administration of antenatal corticosteroids was no longer the optimal strategy.

Univariate sensitivity were performed across a wide range for all model inputs for the latest gestational ages at which corticosteroids resulted in higher combined maternal and infant outcomes. For hospitalized women with COVID-19 at 31 weeks of gestation, the probabilities of ICU admission, maternal death, and neurodevelopmental delay with corticosteroid exposure were most important to our model (-Fig. 2). Antenatal corticosteroids for hospitalized women at high risk of preterm delivery became the less effective management strategy when the probability of maternal ICU admission increased to $31.6 \%$ and the probability of death following an ICU admission increased to $7.7 \%$. When the probability of neurodevelopmental delay with steroid exposure increased to greater than $9.6 \%$, the infant benefits from corticosteroid exposure no longer outweighed the risks to hospitalized women at 31 weeks of gestation. For women admitted to the ICU at 29 weeks of gestation with COVID-19, the probability of maternal death and 


\begin{tabular}{|c|c|c|c|c|}
\hline \multirow{2}{*}{$\begin{array}{l}\text { Gestational } \\
\text { age (wk) }\end{array}$} & \multicolumn{2}{|c|}{ Hospitalized } & \multicolumn{2}{|c|}{ Intensive care unit } \\
\hline & Steroids & $\begin{array}{l}\text { No } \\
\text { steroids }\end{array}$ & Steroids & $\begin{array}{l}\text { No } \\
\text { steroids }\end{array}$ \\
\hline 24 & 407,800 & 358,200 & 389,600 & 345,100 \\
\hline 25 & 460,200 & 424,300 & 442,000 & 411,100 \\
\hline 26 & 492,400 & 462,700 & 474,000 & 449,500 \\
\hline 27 & 516,500 & 496,500 & 497,800 & 483,100 \\
\hline 28 & 531,600 & 519,400 & 512,800 & 505,800 \\
\hline 29 & 539,800 & 533,600 & 520,900 & 519,900 \\
\hline 30 & 547,400 & 545,500 & 528,400 & 531,700 \\
\hline 31 & 551,400 & 549,200 & 532,000 & 535,100 \\
\hline 32 & 559,100 & 559,300 & 539,800 & 545,300 \\
\hline 33 & 564,800 & 565,300 & 565,200 & 551.200 \\
\hline
\end{tabular}

Abbreviations: COVID-19, novel coronavirus disease 2019; QALYs, quality adjusted life years.

neurodevelopmental delay were also important to our model. When the probability of maternal death in the ICU was greater than $7.85 \%$ and the probability of neurodevelopmental delay increased above $12.8 \%$, not using antenatal corticosteroids was the optimal management strategy for women in the ICU at 29 weeks of gestation.

During our multivariable analysis via Monte Carlo simulation, we found that administering corticosteroids was optimal $100 \%$ of the time at 24,25 , and 26 weeks of gestation for women who are hospitalized ( - Table 5 ). For the weeks in which total QALYs were greater without antenatal corticosteroid administration, which were 32 weeks of gestation for hospitalized women and 30 weeks of gestation for women admitted to the ICU, corticosteroid administration was only optimal 43.2 and $25.9 \%$ of the time, respectively. As gestational age increased, the frequency of the time in which corticosteroid administration was effective decreased. In hospitalized patients and women admitted to the ICU at 33 weeks of gestation, corticosteroids were an effective management strategy 0 and $3.4 \%$ of the time, respectively.
Table 5 Frequency of trials antenatal corticosteroid management is optimal in a Monte Carlo simulation of 1,000 hospitalized women and women admitted to the ICU with COVID-19 infection at risk of preterm delivery at 24-33 weeks of gestation

\begin{tabular}{|lll|}
\hline $\begin{array}{l}\text { Gestational } \\
\text { age (wk) }\end{array}$ & $\begin{array}{l}\text { Hospitalized } \\
\text { (\%) }\end{array}$ & $\begin{array}{l}\text { Intensive } \\
\text { care unit (\%) }\end{array}$ \\
24 & 100 & 99.6 \\
25 & 100 & 99.5 \\
26 & 100 & 98.5 \\
27 & 99.3 & 96.5 \\
28 & 97.4 & 83.2 \\
29 & 85.3 & 57.2 \\
30 & 66.2 & 25.9 \\
31 & 65.6 & 20.7 \\
32 & 43.2 & 7.6 \\
33 & 0 & 3.4 \\
\hline
\end{tabular}

Abbreviations: COVID-19, novel coronavirus disease 2019; ICU, intensive care unit.

\section{Discussion}

In this study, we found that antenatal corticosteroid administration for women at risk of preterm delivery with COVID19 infection was an effective management strategy from a combined maternal-infant perspective below 32 weeks of gestation for hospitalized patients and below 30 weeks of gestation for women admitted to the ICU when compared with not administering steroids. In our theoretical cohort, with increasing gestational age at which imminent delivery may occur, steroid administration becomes less effective when both maternal and infant outcomes are considered.

In March, 2020, it was projected that 20 to $60 \%$ of the U.S. population could become infected with COVID-19. ${ }^{27}$ Therefore, with an estimated 3.9 million births in the United States per year, of which $2.76 \%$ are early preterm (less than 34 weeks of gestation), the number of pregnant women with preterm births infected with COVID-19 could be between 60,000 to $190,000 .^{18,26}$ With the high number of women and clinicians encountering this issue, determining the optimal management in this setting is important.
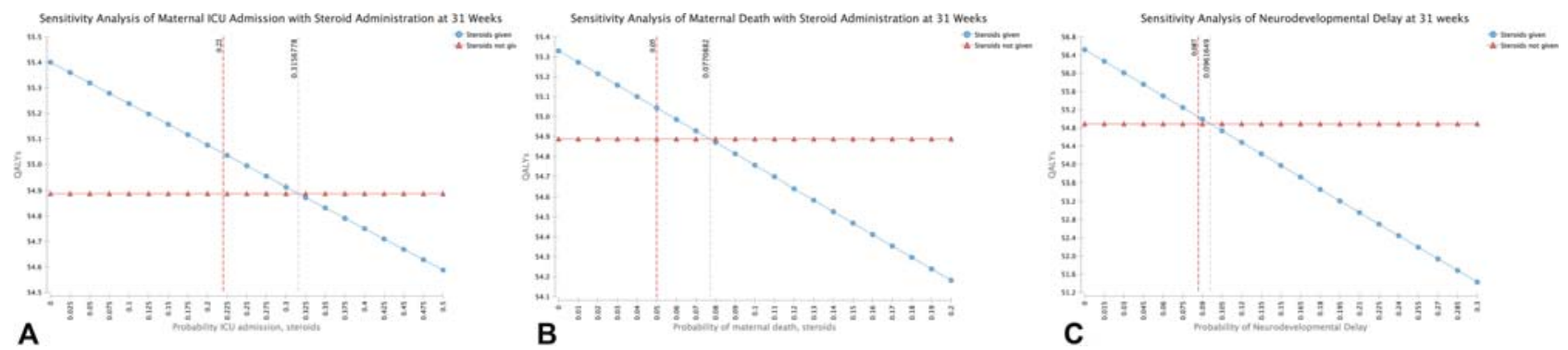

Fig. 2 One-way sensitivity analysis of all hospitalized patients. The $y$-axis represents the QALYs and the x-axis represents the probability of ICU admission (A), maternal death in the ICU (B) and neurodevelopmental delay (C) with corticosteroids use. ICU, intensive care unit; QALYs, quality adjusted life years. 
Our model findings were consistent with published data for previous worldwide viral outbreaks, such as MERS, SARS, and influenza. ${ }^{7,12,28}$ It has been widely demonstrated in the literature that corticosteroid administration in adults with these infections is associated with increased morbidity and mortality, although the exact mechanism is unknown. We found that the probabilities of maternal admission to the ICU and mortality following corticosteroid administration were impactful to our findings during sensitivity analysis. However, it is important to note that the evidence for the effects of corticosteroids in MERS, SARS, and influenza were in the setting of high-dose hydrocortisone corticosteroid treatment, and many of these patients had a more severe disease course. Betamethasone is typically used for antenatal steroid administration. Therefore, it is important that future studies investigate the effects of antenatal corticosteroid on maternal morbidity and mortality during viral outbreaks like COVID-19.

Our model findings were also consistent with published data for the consequences of preterm delivery. We found that the frequency of IVH, neurodevelopmental delay, and infant death were highest at 24 weeks of gestation and decreased as gestational age increased. Similarly, the frequency of each of these adverse outcomes were lower with maternal antenatal corticosteroid administration. Typically, this management strategy would not be questioned, as there are minimal known negative consequences of corticosteroid administration to pregnant women. However, in this setting of COVID19 infection, when the effects of adverse infant and maternal outcomes are balanced, there is a point at which infant benefit is outweighed, such as is seen with expectant management of severe preeclampsia. Additionally, we found that this gestational age tipping point differed for women who were hospitalized versus already in the ICU.

To date, there are no retrospective cohort studies evaluating the effects of antenatal corticosteroids in women with COVID-19 infection and their infants. Mcintosh recently published clinical guidance for corticosteroid use in pregnancy during the COVID-19 pandemic, and her recommendations that steroids should not be used over 32 weeks of gestation, and that decisions should be based on multidisciplinary shared decision making are consistent with our results. While the paper does include data on worsening patient outcomes in COVID-19 with steroid exposure, there are multiple confounders to consider, making the data potentially unreliable. ${ }^{29}$ This is the first decision analysis of its type to investigate the maternal and infant consequences of corticosteroid use during a viral pandemic. The use of decision analysis is an important tool enabling the estimation of clinical implications of corticosteroid administration for both women and infants on a population level, which is a strength of this study as decisions in obstetrics typically are based on a balance of infant and maternal impact.

\section{Limitations}

Decision-analytic models are not without limitations. Models are inherently subject to uncertainty, as model inputs rely on previously published evidence. Despite the quick spread of infection, there is still little evidence on its implications for pregnant women and their infants. ${ }^{30}$ With the current dynamic environment and progression of infection throughout the world, it is important to note that much of this study on the impact of steroids on ICU admission and maternal mortality relied on data from previous influenza studies. Corticosteroid therapy was often used as adjunct therapy for management of SARS, MERS, and influenza to little benefit for patients, but the data for COVID-19 is currently limited. It is also important to acknowledge that corticosteroids used for respiratory syndromes are different than the betamethasone given for fetal lung maturity. Although betamethasone and hydrocortisone are both corticosteroids, they differ in potency, duration of action, and ability to cross the placenta. ${ }^{31}$ Betamethasone is given in two doses of 12-mg intramuscularly 24 hours apart. This would be equivalent to $320 \mathrm{mg}$ of hydrocortisone, which is similar to the dosing given in MERS as reported by Arabi et al of $300 \mathrm{mg}$ daily for a median of 7 days. ${ }^{5,7}$ We also assumed that any woman who died from COVID-19 while pregnant would be treated in the ICU, which was not assumed in the paper by Arabi et al, and it is not necessarily the case in hospitals in the United States that are overwhelmed with the number of COVID-19 cases. Further, many previous studies investigating SARS, MERS, and influenza contained few female participants.

However, we are reassured by our univariate and multivariate analyses that despite a wide range of values in our study for maternal morbidity and mortality following steroids administration, the outcomes do not greatly differ. As the COVID-19 pandemic continues to unfold, further evidence on the impact of antenatal corticosteroids on maternal and infant outcomes may affect which gestational ages that corticosteroid administration should be recommended.

Another limitation was that our cohort of interest was symptomatic hospitalized patients diagnosed with COVID-19 and are at risk of delivery within the next 7 days. Several patients who are hospitalized for clinical management of pregnancy complications, such as preterm prelabor rupture of membrane and preeclampsia, will not deliver within the week. ${ }^{32}$ Our model does not factor in the population at low risk for imminent preterm delivery and the latency until delivery following presentation. As COVID-19 lasts approximately 2 weeks in a majority of patients, if a woman continues to be pregnant for 2 weeks or greater following initial presentation, she potentially no longer has the associated risks of steroid exposure in the setting of an infection. Further, there are many more asymptomatic COVID-19 positive patients than symptomatic, thus this model is only applicable to symptomatic patients at high risk for preterm delivery. ${ }^{33}$ COVID-19 test results should not be a prerequisite for administration of antenatal steroids to an asymptomatic patient at risk for pre-term delivery.

\section{Conclusion}

In conclusion, our findings suggest that antenatal corticosteroid administration for women at high risk of preterm delivery with a COVID-19 infection have improved combined maternal and infant outcomes below 32 weeks of gestation 
for hospitalized patients and below 30 weeks of gestation for patients admitted to the ICU. As decisions regarding care of women and infants in the preterm period are complex, the risks, benefits, and alternatives should be considered during conversations with patients. This decision analysis was conducted because of the paucity of data informing the use of antenatal corticosteroids for preterm delivery in COVID-19positive patients. Thus, care of women with respiratory compromise from COVID-19 should be individualized, and such patients should receive multidisciplinary team care, with MFM and critical care specialists. The results of the study can guide management with the clinical dilemma of weighing maternal risks to infant benefits in the preterm period of pregnant women with COVID-19 infection.

Funding

None.

Conflict of Interest

None declared.

\section{References}

1 CDC. If You Are Pregnant, Breastfeeding, or Caring for Young Children. Available at: https://www.cdc.gov/coronavirus/2019ncov/hcp/pregnant-women-faq.html\#pregnant. Accessed May 20, 2020

2 World Health Organization. Clinical management of severe acute respiratory infection when COVID-19 disease is suspected. Available at: https://www.who.int/publications-detail/clinical-management-of-severe-acute-respiratory-infection-when-novel-coronavirus-(ncov)-infection-is-suspected. Accessed May 20, 2020

3 Rasmussen SA, Smulian JC, Lednicky JA, Wen TS, Jamieson DJ. Coronavirus Disease 2019 (COVID-19) and pregnancy: what obstetricians need to know. Am J Obstet Gynecol 2020;222(05):415-426

4 Guan W-J, Ni Z-Y, Hu Y, et al; China Medical Treatment Expert Group for Covid-19. Clinical characteristics of coronavirus disease 2019 in China. N Engl J Med 2020;382(18):1708-1720. Doi: 10.1056/NEJMoa2002032

5 Roberts D, Dalziel S. Antenatal corticosteroids for accelerating fetal lung maturation for women at risk of preterm birth. Available at: http://onlinelibrary.wiley.com/doi/10.1002/146518 58.CD004454.pub2/pdf. Accessed May 20, 2020

6 Russell CD, Millar JE, Baillie JK. Clinical evidence does not support corticosteroid treatment for 2019-nCoV lung injury. Lancet 2020; 395(10223):473-475

7 Arabi YM, Mandourah Y, Al-Hameed F, et al; Saudi Critical Care Trial Group. Corticosteroid therapy for critically ill patients with middle east respiratory syndrome. Am J Respir Crit Care Med 2018;197(06):757-767

8 Longman RE, Johnson TRB. Viral respiratory disease in pregnancy. Curr Opin Obstet Gynecol 2007;19(02):120-125

9 Lee N, Allen Chan KC, Hui DS, et al. Effects of early corticosteroid treatment on plasma SARS-associated Coronavirus RNA concentrations in adult patients. J Clin Virol 2004;31(04):304-309

10 Auyeung TW, Lee JSW, Lai WK, et al. The use of corticosteroid as treatment in SARS was associated with adverse outcomes: a retrospective cohort study. J Infect 2005;51(02):98-102

11 CDC COVID-19 Response Team. Severe outcomes among patients with coronavirus disease 2019 (COVID-19) - United States, February 12-March 16, 2020. MMWR Morb Mortal Wkly Rep 2020; 69(12):343-346

12 Lansbury LE, Rodrigo C, Leonardi-Bee J, Nguyen-Van-Tam J, Shen Lim W. Corticosteroids as adjunctive therapy in the treatment of influenza: an updated cochrane systematic review and metaanalysis. Crit Care Med 2020;48(02):e98-e106

13 Travers CP, Clark RH, Spitzer AR, Das A, Garite TJ, Carlo WA. Exposure to any antenatal corticosteroids and outcomes in preterm infants by gestational age: prospective cohort study. BMJ 2017;356(j1039):j1039

14 Larroque B, Ancel P-Y, Marret S, et al; EPIPAGE Study group. Neurodevelopmental disabilities and special care of 5-year-old children born before 33 weeks of gestation (the EPIPAGE study): a longitudinal cohort study. Lancet 2008;371(9615):813-820

15 Mukerji A, Shah V, Shah PS. Periventricular/intraventricular hemorrhage and neurodevelopmental outcomes: a meta-analysis. Pediatrics 2015;136(06):1132-1143

16 Sanders GD, Neumann PJ, Basu A, et al. Recommendations for conduct, methodological practices, and reporting of cost-effectiveness analyses: Second panel on cost-effectiveness in health and medicine. JAMA 2016;316(10):1093-1103

17 Team IS; InterLACE Study Team. Variations in reproductive events across life: a pooled analysis of data from 505147 women across 10 countries. Hum Reprod 2019;34(05):881-893

18 Martin JA, Hamilton BE, Osterman MJK, Driscoll AK, Drake P. Births: final data for 2017. Natl Vital Stat Rep 2018;67(08):1-50

19 Blair E, Watson L, Badawi N, Stanley FJ. Life expectancy among people with cerebral palsy in Western Australia. Dev Med Child Neurol 2001;43(08):508-515

20 WHO. Annex 2: tables of health statistics by country, WHO region and globally. Available at: https://www.who.int/gho/publications/ world_health_statistics/2019/EN_WHS_2019_Annex2.pdf?ua=1. Accessed May 20, 2020

21 Day SM, Reynolds RJ, Kush SJ. Extrapolating published survival curves to obtain evidence-based estimates of life expectancy in cerebral palsy. Dev Med Child Neurol 2015;57(12):1105-1118

22 Cuthbertson BH, Roughton S, Jenkinson D, Maclennan G, Vale L. Quality of life in the five years after intensive care: a cohort study. Crit Care 2010;14(01):R6

23 Carroll AE, Downs SM. Improving decision analyses: parent preferences (utility values) for pediatric health outcomes. J Pediatr 2009; 155(01):21-25, 25.e1-25.e5

24 Kuppermann M, Nease RF, Learman LA, Gates E, Blumberg B, Washington AE. Procedure-related miscarriages and Down syndrome-affected births: implications for prenatal testing based on women's preferences. Obstet Gynecol 2000;96(04):511-516

25 Saigal S, Stoskopf B, Pinelli J, et al. Self-perceived health-related quality of life of former extremely low birth weight infants at young adulthood. Pediatrics 2006;118(03):1140-1148

26 Phibbs CS, Schmitt SK. Estimates of the cost and length of stay changes that can be attributed to one-week increases in gestational age for premature infants. Early Hum Dev 2006;82(02):85-95

27 Tsai TC, Jacobson BH, Jha AK. American hospital capacity and projected need for COVID-19 patient care. Available at: https:// www.healthaffairs.org/do/10.1377/hblog20200317.457910/full/. Accessed May 20, 2020

28 Hui DSC, Chan PKS. Severe acute respiratory syndrome and coronavirus. Infect Dis Clin North Am 2010;24(03):619-638

29 McIntosh JJ. Corticosteroid guidance for pregnancy during COVID19 pandemic. Am J Perinatol 2020;2019:1-4

30 Dotters-Katz S, Hughes B; Society for Maternal fetal medicine. Coronavirus (COVID-19) and pregnancy: what maternal-fetal medicine subspecialists need to know. Available at: https://s3. amazonaws.com/cdn.smfm.org/media/2262/COVID19_PDF.pdf. Accessed May 20, 2020

31 Moore LE, Martin JN Jr. When betamethasone and dexamethasone are unavailable: hydrocortisone. J Perinatol 2001;21(07):456-458

32 Practice Bulletin No. Practice bulletin no. 171: management of preterm labor. Obstet Gynecol 2016;128(04):e155-e164

33 Sutton D, Fuchs K, D'Alton M, Goffman D. Universal screening for SARS-CoV-2 in women admitted for delivery. N Engl J Med 2020;382(22):2163-2164 